\title{
Gold standard treatment of the patients with total knee arthroplasty infection
}

\author{
Kaan YÜKSEL, Kasım KILIÇARSLAN, Mahmut N. AYTEKIN, Mahmut UĞURLU, Nihat TOSUN
}

Department of Orthopedics and Traumatology, Ankara Yıldırım Beyazıt University, Atatürk Education and Research Hospital, Ankara, Turkey

ABSTRACT

This study aimed to evaluate and compare early and mid-term results of the two-stage revision method used for diagnosing infections and giving treatment to patients who underwent total knee arthroplasty in Ankara Atatürk Research and Training Hospital, Turkey, between October 2013 and October 2015.

Patient files were examined retrospectively, and patients were called for the last examination. Further, 23 patients were included in the study. The first and second stages of the two-stage revision were performed for all these patients. However, in 2 of these 23 patients, the process ended with arthrodesis and amputation. Infections were diagnosed based on the clinical examination of the knee, erythrocyte sedimentation rate, C-reactive protein, white blood cell counts, and intraoperative culture results. Laboratory parameters were also used for monitoring infection. Patients were evaluated preoperatively and postoperatively according to the American Knee Society clinical and functional scoring system.

The mean age of the patients was 66.8 years. Also, 18 of the patients were female and 5 male. Early infection was detected in 4, delayed infection in 7, and late infection in 12 patients. The average follow-up time was 12.6 months. The American Knee Society clinical score was 35.0 (median score) preoperatively and 75.4 (median score) postoperatively. The functional score was 38.8 (median score) preoperatively and 77.3 (median score) postoperatively.

In conclusion, early and mid-term results of patients with infected total knee prosthesis, who underwent the two-stage revision, were found to be consistent with the published results in terms of infection eradication success, radiological findings, and postoperative clinical and functional scores. It is possible to increase the success rate by approaching the cases in a standardized and systematic way.

Key words: Knee joint, infected total knee arthroplasty, two-stage revision

\section{INTRODUCTION}

The life expectancy has increased in today's world. As a result, the incidence of articular pathologies such as arthritis has increased too. Also, rheumatic diseases are more common than in the past. That is why primary joint arthroplasties and revision surgeries are increasingly performed these days (1).

The most common cause of total knee arthroplasty revisions is an infection. The possibility of infection in total knee prosthesis varies between $0.4 \%$ and $4.0 \%$ (2). The approach to infected knee replacement includes various treatment methods such as suppression with antibiotics, debridement, arthrodesis, amputation, and single-stage revision and twostage revision surgeries (3). Of these methods, suppression with antibiotics can only be applied to the patients with a bad general condition who are unable to receive anesthesia. Antibiotic therapy using the debridement method is used particularly in patients diagnosed within the first 4 weeks after arthroplasty because bacterial colonization roots after 4 weeks on average. Previous studies have reported up to $77 \%$ treatment success using this method in an early stage $(4,5)$.

Correspondence:

Kaan YÜKSEL

Ankara Yıldırım Beyazıt Üniversitesi Atatürk Eğitim ve Araştırma Hastanesi, Ortopedi ve Travmatoloji Bölümü, Ankara, Türkiye. e-mail: kaanyukselim@hotmail.com 
Arthrodesis and amputation are not widely accepted methods nowadays. However, they are used in patients developing skin problems, patients with additional diseases, patients with inadequate bone stock, and patients with extensor mechanism problem (6).

Single-stage and two-stage revision surgeries are the methods with the best functional and clinical results among the aforementioned methods. One-stage revision means removal of infected prosthesis, surgical debridement, and placement of new prosthesis in the same surgery. However, in the two-stage revision, antibiotic suppression is performed in the first surgery and prosthesis is added in the second surgery.

The present study was conducted to evaluate the clinical and radiological effects of the two-stage revision performed on patients with infected knee arthroplasty and compare the results with published findings.

\section{MATERIALS AND METHODS}

Patients diagnosed with infected knee arthroplasty who decided to undergo the two-stage revision in Orthopedics and Traumatology Department, Ankara Atatürk Training and Research Hospital between October 2013 and October 2015 were selected. Finally, 23 patients were included in the study. The study aimed to deliver early and mid-term results through a 24-month follow-up with the patient following up for the longest duration. The records of patients meeting the study inclusion criteria were analyzed retrospectively. They were contacted again, and the last clinical examination, laboratory results, radiographies, and knee score surveys were renewed.

Anteroposterior (AP) and lateral radiographs were obtained from all these patients before the first stage, between the first and second stages, and after the second stage. Findings on loosening were analyzed, and alpha, beta, gamma, and sigma angles were evaluated according to the American Knee Society's radiological evaluation form in terms of proper settlement of components (7). All patients filled in the American Knee Society clinical and functional knee score questionnaire (8).
Clinically, erythema, pain, and motion were assessed. If fistula opening was observed on the skin, the situation was recorded. The erythrocyte sedimentation rate (ESR), C-reactive protein (CRP) level, complete blood count, and white blood cell count (WBC) were obtained. Further, culture results were obtained from samples of patients who underwent joint aspiration.

Existing prostheses were removed during the first phase in all patients, debridement was performed, and samples were taken for culture. A spacer with antibiotics (gentamycin) or cement with antibiotics (gentamycin/vancomycin) tailored for the knee was placed inside. Hemovac drain was placed, lines were closed duly, and Robert Jones bandage was applied. The drain was removed on postoperative day 2, and sutures were removed on day 15. Upon the recommendation of Infectious Diseases Department, antibiotics were applied for 6 weeks (intravenously in the first 4 weeks and orally in the next 2 weeks). The patients were called for the follow-up in second, fourth, and sixth weeks postoperatively for evaluating laboratory parameters. Laboratory findings and clinical status were taken into account while planning for the second stage after 10 days without antibiotics. The patients with improved clinical status and laboratory parameters were moved for the second stage and underwent a new prosthesis placement.

The Student t test was used for statistically analyzing the data, and a P value less than 0.05 was considered significant.

\section{RESULTS}

A total of 23 patients were included in the study. All patients underwent the first stage involving the removal of infected material, debridement, and application of an antibiotic spacer or antibiotic cement. The second stage, which consisted of the revision of knee prosthesis, was successfully applied in 21 patients. The revision was not successful in two patients. In one of these patients, the prosthesis was removed due to re-infection in the early stage, and debridement and antibiotic treatment were applied. Next, the arthrodesis procedure was performed on the patient's request. The other patient had already undergone two surgeries, first being primary total knee arthrodesis and second being revision total knee prosthesis. Serious wound site 


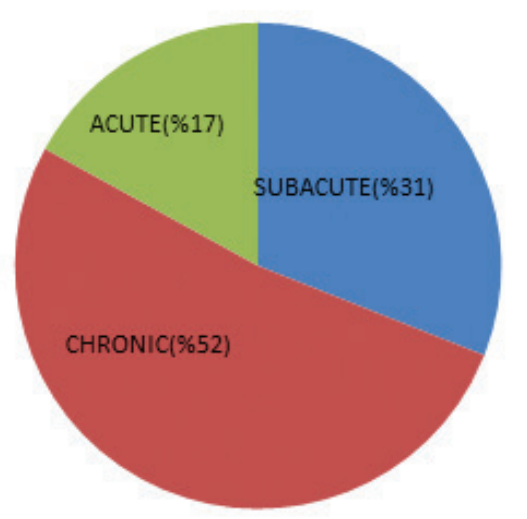

FIgURE 1: Patient classification according to onset.

problems occurred after the first and second stages of revision surgeries. Also, fungal infection (non-albicans Candida) was observed throughout the process. The side effects of antibiotics and antifungal drugs affected the vital functions of the patient. This was discussed with the patient and relatives, and a decision to perform knee top amputation was made mutually. Data from these two patients were not included while presenting statistical values after the second stage of the study.

In 2 of 23 patients, the sinus tract was visible at the time of application. Again, two of the patients had a history of total knee arthroplasty.

A number of classifications have been proposed for infections developing after total knee arthroplasty. The most widely accepted classification is the one proposed by Schofroth and Zimmerli based on the timing of infection. The infection is classified as early (acute) if it develops within the first 3 months, subacute (delayed) if it develops between 3 months and 24 months, and late (chronic-hematogenous) if develops after 24 months. According to this classification, 4 (17\%) of our patients had early (acute), 7 (31\%) had delayed (subacute). and the remaining 12 (52\%) had late (chronic-hematogenous) infections. The patient classification according to onset is shown in Figure 1.

The average and median ages at diagnosis of infection in patients of all groups were found to be 66.8 years [standard deviation (SD) $8.0]$ and 67 years (minimum 49 and maximum 80), respectively.
In males, they were 69.6 years (SD 8.6) and 68 years (minimum 61 and maximum 80), respectively; in females, they were 66.2 (SD 7.9) and 67 years (minimum 49 and maximum 81), respectively. Further, 18 of these 23 patients were female and 5 were male. Six complained of infection in the left knee and 17 in the right knee.

The average and median times between the first and second stages of surgery in both groups were 66.4 days (SD 15.7) and 67.5 days (minimum 43 to maximum 95), respectively. The average and median periods were 66.4 days (SD 15.7) and 68.5 days (minimum 56 and maximum 80) in male patients and 65.9 days (SD 16.7) and 67.5 days (minimum 42 and maximum 80) in female patients, respectively. The data are shown in Figure 2.

This time difference between the two sexes was not statistically significant $(P>0.05)$. However, this period was much longer than 6 weeks, which is generally accepted according to the published studies. The reasons were as follows: (1) most patients had no culture growth, and hence the eradication of infection was not certain due to empirical antibiotics treatment, (2) their laboratory findings and clinical presentation did not prove the eradication of infection; and patients were unable to come to the hospital at desired times.

Before applying spacer, the mean ESR value of patients was 79.7 $\mathrm{mm} / \mathrm{h}$ (SD 30.1), pre-revision mean ESR value was $36.2 \mathrm{~mm} / \mathrm{h}$ (SD 12.9) and the mean ESR value at the end of follow-up was $39.3 \mathrm{~mm} / \mathrm{h}$ (SD 11.7). When the pre-spacer and pre-revision ESR 


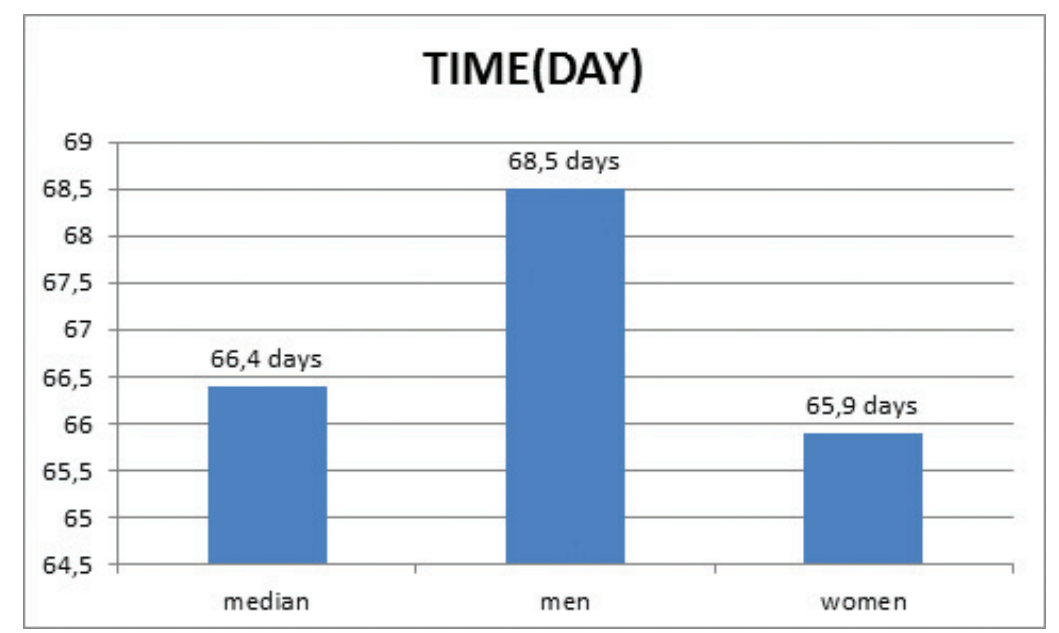

FIGURE 2: Time between the first and second stages of surgery (in days).

averages were compared, a statistically significant difference was found $(P<0.05)$. However, no statistically significant difference was observed between pre-revision ESR and post-follow-up ESR $(P>0.05)$.

The average CRP value of patients prior to the application of the spacer was $34.6 \mathrm{mg} / \mathrm{dL}$ (SD 21.7). When the same parameter was observed in terms of pre-revision values and follow-up values, it was found to be $4.7 \mathrm{mg} / \mathrm{L}$ (SD 3.0) and $4.2 \mathrm{mg} / \mathrm{L}$ (SD 2.1), respectively. When these values were compared statistically, the lower value of pre-revision CRP compared with pre-spacer CRP was found to be significant with $P<0.05$. However, when the follow-up CRP levels were compared with pre-revision CRP levels, no statistically significant difference was found ( $P>0.05)$.

While the pre-spacer WBC value of patients was $7.78 / \mathrm{mm}^{3}$ (SD 2.3), the pre-revision WBC value was $7.24 / \mathrm{mm}^{3}$ (SD 2.3). The follow-up WBC value was $7.29 / \mathrm{mm}^{3}$ (SD 2.0). Comparison of these results revealed that pre-spacer, pre-second stage, and post-revision follow-up WBC values did not change in a statistically significant manner $(P>0.05)$.

Table 1 shows us laboratory values of patients at various stages of two-stage revision surgery (ESR, (RP, WBC).

The components pre- and postoperatively were compared according to the American Knee Society's radiographic evaluation form (7). Statistically significant differences were not observed $(P>0.05)$.

The American Knee Society clinical and functional scores (8) were determined preoperatively and postoperatively. The average preoperative and postoperative knee scores were 35.0 (SD 5.2) and 75.4 (SD 10.2), respectively. These data were compared, and a statistically significant result was obtained $(P<0.05)$. Similarly, when functional scores were compared, the preoperative and postoperative values were found to be

TABLE 1: Laboratory values of patients at various stages of two-stage revision surgery.

\begin{tabular}{llll}
\hline & ESR (mean) & CRP (mean) & WBC (mean) \\
\hline Before first stage & $79.7 \mathrm{~mm} / \mathrm{h}$ (SD 30.1) & $34.6 \mathrm{mg} / \mathrm{L}$ (SD 21,7) & $7.78 / \mathrm{mm}^{3}(\mathrm{SD} 2.3)$ \\
Before second stage & $36.2 \mathrm{~mm} / \mathrm{h}$ (SD 12.9) & $4.7 \mathrm{mg} / \mathrm{L}(\mathrm{SD} 3,0)$ & $7.24 / \mathrm{mm}^{3}(\mathrm{SD} 2.3)$ \\
Post-revision follow-up & $39.3 \mathrm{~mm} / \mathrm{h}$ (SD 11.7) & $4.2 \mathrm{mg} / \mathrm{L}(\mathrm{SD} 2,1)$ & $7.29 / \mathrm{mm}^{3}(\mathrm{SD} 2.0)$ \\
$\begin{array}{l}\text { Statistical difference between before } \\
\text { and after revision values }\end{array}$ & $\mathrm{P}<0.05$ & $\mathrm{P}<0.05$ & $\mathrm{P}>0.05$ \\
\hline
\end{tabular}


TABLE 2: Patient evaluation according to the American Knee Society clinical and functional scoring system.

\begin{tabular}{lll}
\hline & Clinical score (mean) & Functional score (mean) \\
\hline Preoperative stage & 35 & 38.8 \\
Postoperative stage & 75.4 & 77.3 \\
\hline
\end{tabular}

38.8 (SD 9.7) and 77.3 (SD 10.2), respectively. These data were compared, and a statistically significant result was obtained ( $P$ $<0.05$ ). We see this evaluation in Table 2.

Figure 3 shows the roentgenogram of one of the patients in this study. This patient was a 75 -year-old male. His first surgery (total knee arthroplasty) was performed 5 years ago.

Figure 4 shows the first-stage surgery roentgenogram after infection.

Figure 5 shows the roentgenogram of the patient after the secondstage surgery.

\section{DISCUSSION}

Total knee arthroplasty revision surgeries are increasingly performed nowadays. Infections constitute the most common cause of revision surgeries $(1,3)$. The single-stage revision method to treat these patients is being less preferred these days because resistant microorganisms, such as methicillin-resistant bacteria, are seen more often, especially in the last 20 years (10). Better clinical and laboratory observations and antibiotherapy prior to the second stage made the two-stage revision the gold standard method of approach.

Care should be taken during the diagnosis of these patients. Physical examination and laboratory parameters are especially important. Local tenderness, erythema, increased temperature, fever, and sinus tract presence are important physical examination parameters. The laboratory parameters include ESR, CRP, and WBC. The increase in ESR and CRP is normal after the surgery. However, CRP returns to normal in 1-2 days, although ESR returns to normal in 2-3 months. The quick return of CRP value compared with ESR is important. Therefore, CRP is a more important marker
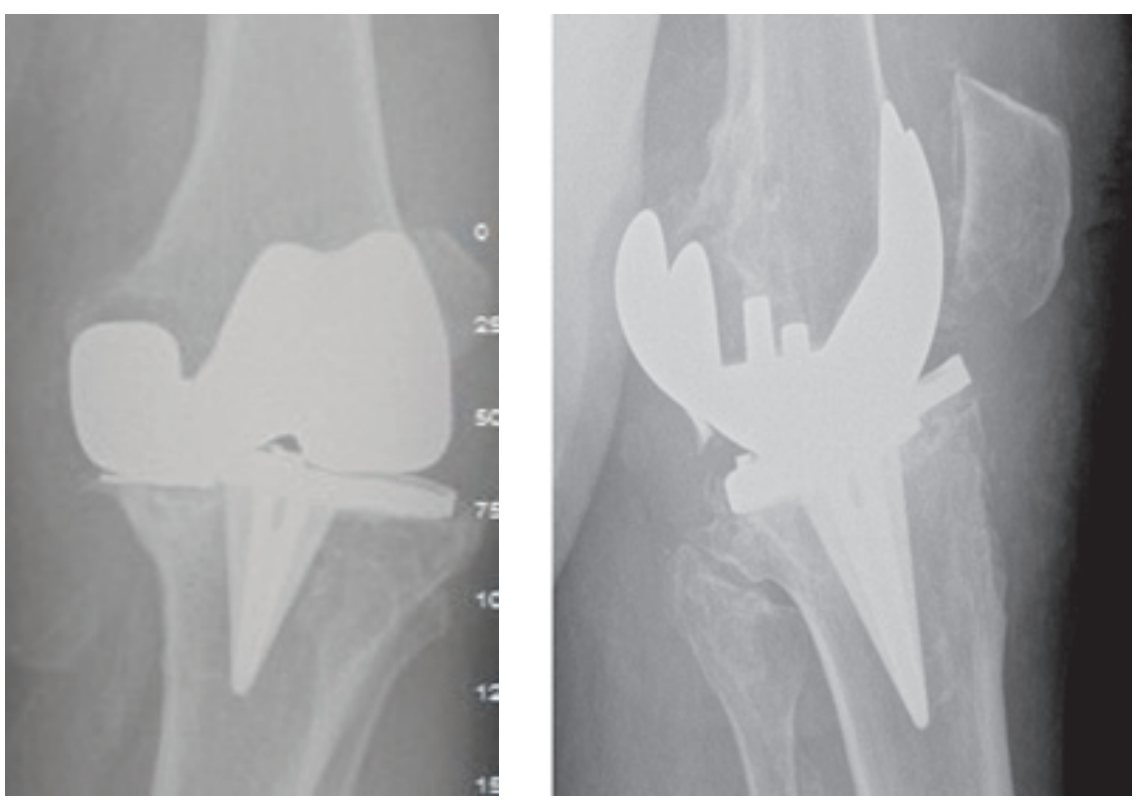

FIGURE 3: Roentogram of a 75-year-old male patient who underwent total knee arthroplasty 5 years ago. 

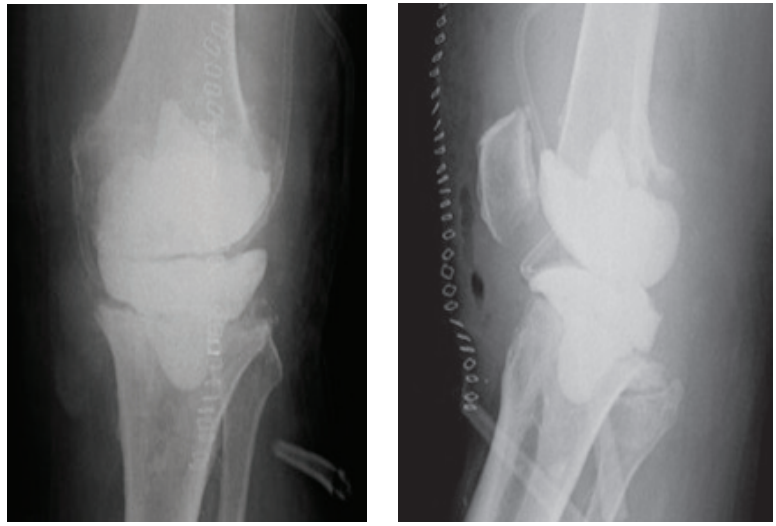

FIGURE 4: Roentgenogram after the first-stage surgery.
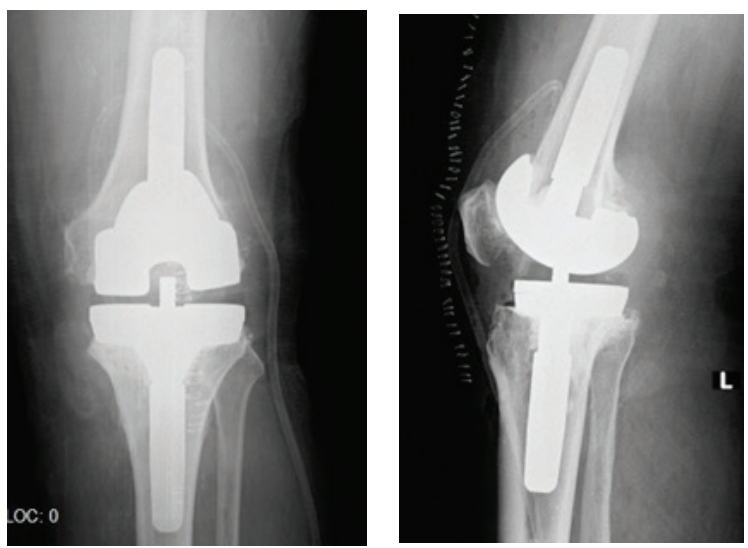

FIGURE 5: Roentgenogram after the second-stage surgery. of infection follow-up $(11,12)$. In the present study, a statistically significant difference was found in ESR and CRP between diagnosis and first- and second-revision surgery stages. However, no statistically significant difference was found in WBC count. Several studies showed that CRP was valuable in diagnosis and follow-up of infection, ESR helped in evaluating CRP, and WBC count should not be used in this process (13).

The gold standard while diagnosing infection in patients undergoing total knee arthroplasty is to take culture samples from at least three different areas of the deep tissue from. In addition, inoculating from the fluid in the knee intraoperatively increases the chances of diagnosis (14).

Despite using all these methods, causative organisms were found in only 13 (56\%) of patients. In published studies, this rate has been stated as 65\%-94\% $(13,14)$. Microorganisms were found in fewer patients compared with published data due to many reasons. The main reason was patients' search for many outside centers and uncontrolled antibiotherapy application in these centers, leading to the development of resistant microorganisms. Also, in recent years, methicillin-resistant bacteria are seen more frequently as causative microorganisms in infected patients undergoing total knee arthroplasty. Inappropriate antibiotics use is also one of the reasons. It has led to some difficulties in diagnosis and treatment (16).

In addition, some studies proposed routine preoperative synovial fluid aspiration culture, while others proposed no need for the culture $(17,18)$. Routine preoperative knee aspiration was not performed in the department where the present study was conducted.

The antibiotherapy length between two stages is an important parameter. A large-scale study published in 2006 explored adequate and inadequate antibiotherapy. It showed that 6 weeks of antibiotherapy with at least 2 weeks of IV administration was adequate antibiotherapy (19). This method was used in the present study.

The two-stage revision is the gold standard in methods used for treating infected knee prosthesis. This method is a very effective method in improving functional limitations that affect daily lives of patients and eradicating infection, and hence should be preferred $(3,20)$. The present study supported the use of the aforementioned method.

\section{REFERENCES}

1. Insall JN, Thompson FM, Brause BD (1983) Two-stage reimplantation for the salvage of infected total knee arthroplasty. J Bone Joint Surg Am 65(8):1087-1098

2. Bozic KJ, Kurtz SM, Lau E, et al. The epidemiology of revision TKA in the United States. Clin Orthop Relat Res 2010;468:45.

3. Haleem AA, Berry DJ, Hanssen AD. Mid-term to long-term followup of two-stage reimplantation for infected TKA. Clin Orthop Relat Res 2004;428:35.

4. Segawa H, Tsukayama DT, Kyle RF, et al. Infection after total knee arthroplasty. A retrospective study of the treatment of eighty-one infections. J Bone Joint Surg Am 1999;81:1434. 
5. Van Kleunen JP, Knox D,Garino JP, et al. Irrigation and debridement and prosthesis retention for treating acute periprosthetic infections. Clin Orthop Relat Res 2010;468(8):2024

6. Rand JA. Evaluation and management of infected total knee arthroplasty. Seminars in Arthroplasty 5(4): 1994, 178

7. Frederick C. The Knee Society Total Knee Arthroplasty Roentgenographic Evaluation and Scoring System. Clin. Orthop. March 28, 1989; 9-13

8. Insall J, Dorr L, Scott R. Rationale of The Knee Society Clinical Rating System. Clin Orthop March 28, 1989; 13-14

9. Schofroth $M$, Zimmerli W. İfections. Ochsner PE ed. Total hip replacement. Berlin, Springer Verlog 2003; 65-90

10. Gallo J, Kolár M, Koukalová D, et al. Bacterial pathogens of periprosthetic infections and diagnostic possibilities. Klin Mikrobiol Infekc Lek 2006;12:117-23 (in Czech).

11. Tetsworth K. İnfection after total knee arthroplasty: evaluation and treatment. Current Opinion in orthopaedics. 14, 2003; 45-51

12. Niskanen RO et all. Serum C-reaktive protein levels after total hip and knee arthroplasty. JBJS. 78 B, 1996; 431-436

13. Claassen I. Preoperative diagnostic for periprosthetic joint infection prior to total knee revision arthroplasty. 2014; 6: 5437.
14. Zimmerli W. Prosthetic joint associated infections. Best Pract Res Clin Rheumatol 2006; 20: 1045-1063.

15. Trampuz A. Widmer AF. İnfections associated with orthopaedic implants. Curr Opin Infect Dis 2006: 19;349-356

16. Parvizi J, Bender B, Saleh KJ, et al. Resistant organisms in infected total knee arthroplasty: occurrence, prevention, and treatment regimens. Instr Course Lect 2009;58:271-278.

17. Parvizi J, Jacovides C, Zmistowski B, Jung KA. Definition of periprosthetic joint infection: is there a consensus? Clin Orthop Relat Res 2011;469:3022-30.

18. Durbhakula SM. Antibiotic loaded articulating cement spacer in the 2 stage Exchange of infected total knee arthroplasty. The Journal of Arthroplasty. 2004: 19; 768-774

19. Laffer RR. Outcome of prosthetic knee associated infection: evaluation of consecutive episodes at a single center. Clin Microbiol Infect 2006: 12; 433-439

20. Leone S, Borre S, Monforte A, et al. Consensus document on controversial issues in the diagnosis and treatment of PJIs. Int $J$ Infect Dis 2010;14(Suppl 4):S67. 